8. Kono H, Rock KL. How dying cells alert the immune system to danger. Nat Rev Immunol 2008; $8: 279-89$.

9. Gilliet M, Cao W, Liu YJ. Plasmacytoid dendritic cells: sensing nucleic acids in viral infection and autoimmune diseases. Nat Rev Immunol 2008 ; $8: 594-606$.
10. Banchereau J, Pascual V. Type I interferon in systemic lupus erythematosus and other autoimmune diseases. Immunity 2006 ; 25 : 383-92

11. Angelot F, Seilles $\varepsilon$, Biichle $S$, et al. Endothelial cellderived microparticles induce plasmacytoid dendritic cell maturation: potential implications in inflammatory diseases. Haematologica 2009 ; 94 : 1502-12.
12. Simoncini S, Njock MS, Robert S, et al. TRAIL/Apo2L mediates the release of procoagulant endothelial microparticles induced by thrombin in vitro: a potential mechanism linking inflammation and coagulation. Circ Res 2009; 104 : 943-51.

\title{
NOUVELLE
}

\section{Clathrine CHC22, trafic intracellulaire de GLUT4 et diabète de type 2}

Stéphane Vassilopoulos, Christopher Esk, Sachiko Hoshino, Frances M. Brodsky
Department of bioengineering and therapeutic sciences, University of California, School of Pharmacy, San Francisco (UCSF), 513 Parnassus avenue, HSW 1526-1528, Box 0552 University of California, San Francisco CA 94143-0552, États-Unis. stephane.vassilopoulos@upmc.fr
> Chez l'homme, le tissu musculaire et le tissu adipeux sont majoritairement responsables de l'absorption du glucose sanguin en réponse à l'insuline [1]. Les cellules qui composent ces tissus possèdent, en plus des transporteurs ubiquitaires GLUTl [14], un grand nombre de transporteurs, les GLUT4, qui se caractérisent principalement par leur localisation dans des compartiments intracellulaires. L'insuline qui est sécrétée par les îlots pancréatiques après un repas induit une augmentation de GLUT4 à la surface des cellules; ce qui permet la diminution des taux sanguins de glucose. Chez les patients atteints de diabète de type 2, l'insuline n'est plus capable d'induire la capture du glucose sanguin par les cellules musculaires [2, 3]. Notre équipe a identifié une protéine qui joue un rôle important dans le trafic membranaire de GLUT4 chez l'homme et dont l'expression est perturbée chez les patients atteints de diabète de type 2 .

\section{Notion de vésicules}

de stockage de GLUT4

Les mécanismes qui permettent la présence des transporteurs du glucose à la surface de la cellule sont complexes et ont fait l'objet de nombreuses études dans les vingt dernières années.
En conditions basales, c'est-à-dire en l'absence d'insuline, seuls les transporteurs GLUTl, présents sur la membrane des cellules musculaires et adipeuses, assurent l'entrée du glucose nécessaire au métabolisme cellulaire. GLUT4 est lui séquestré dans un compartiment intracellulaire, limitant l'entrée du glucose dans la cellule. L'augmentation de la concentration d'insuline va spécifiquement déclencher la translocation des vésicules et l'insertion des transporteurs du glucose à la surface cellulaire afin de faciliter l'absorption du glucose. Dans un second temps, GLUT4 est internalisé par endocytose puis se retrouve au niveau des endosomes (Figure 1) [4]. Contrairement à la majorité des protéines de surface qui sont tout de suite recyclées vers la membrane plasmique, GLUT4 est alors adressé vers une population de vésicules de stockage en attendant de migrer vers la surface de la cellule lors d'une nouvelle stimulation par l'insuline.

\section{Deux isoformes de la chaîne lourde} de la clathrine chez l'humain La clathrine est une protéine constituant un manteau (de l'anglais coat) autour des vésicules impliquées dans le transport de protéines entre la mem- brane plasmique et des compartiments intracellulaires comme le réseau transgolgien (trans-golgi network ou TGN) et les endosomes. Elle facilite de façon sélective la formation de ces vésicules. II existe deux isoformes de la chaîne lourde (HC pour heavy chain) de la clathrine chez l'homme, chacune portant le nom du chromosome humain qui contient son gène. $\mathrm{CHCl} 7$ est l'isoforme ubiquitairement exprimée; elle participe à l'endocytose, à la biogenèse des lysosomes ainsi qu'au trafic dans la voie sécrétoire à partir du TGN dans tous les types cellulaires. Notre travail a porté sur le rôle de $\mathrm{CHC} 22$, une nouvelle isoforme de la chaîne lourde de la clathrine, dans le transport de GLUT4. Chez l'homme, CHC22, codée par le chromosome 22, est fortement exprimée dans le muscle squelettique [5]. Cependant, chez la souris, le gène codant pour $\mathrm{CHC} 22$ est un pseudogène [6]. Nous avons montré que, contrairement à $\mathrm{CHCl} 7$ qui, dans les cellules musculaires, intervient dans l'endocytose de GLUT4 en s'associant à la protéine adaptatrice AP2, CHC22 est associée à des vésicules qui contiennent des protéines importantes pour le tri de GLUT4 à partir des endosomes, du TGN et vers les vésicules de stockage (Figure 1). Ces protéines, les protéines 


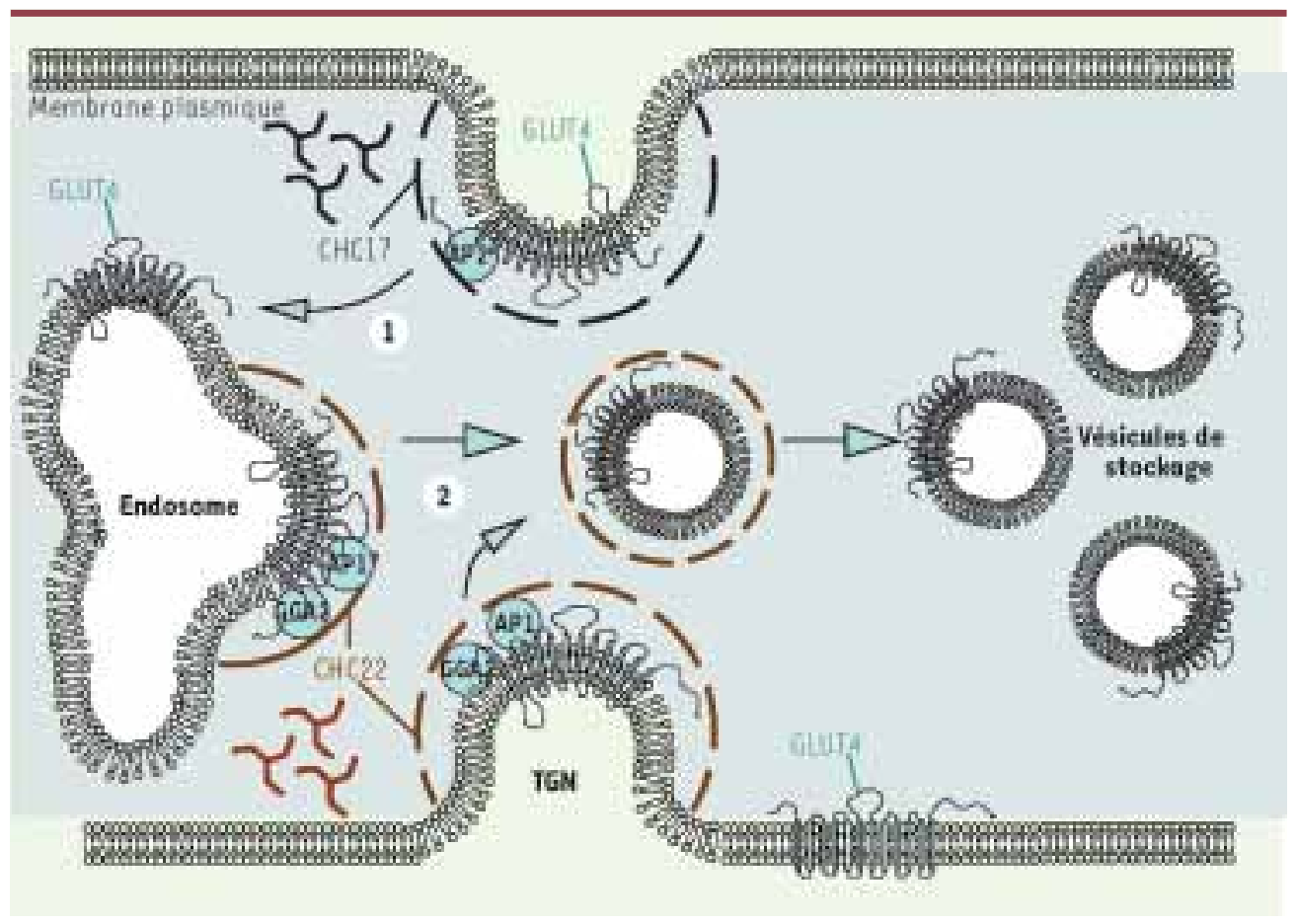

Figure 1. Intervention de CHC17 et CHC22 dans le trafic de GLUT4 chez l'humain. (1) La clathrine $\mathrm{CHCl7}$ (noir pointillé) ainsi que la protéine adaptatrice AP2 participent à l'endocytose de GLUT4 à partir de la membrane plasmique. (2) La clathrine CHC22 (brun pointillé) participe au trafic intracellulaire de GLUT4. En interagissant avec les protéines adaptatrices APl et GGA2, CHC22 permet l'adressage de GLUT4 à partir des endosomes ou à partir du réseau transgolgien (TGN) vers le compartiment de stockage intracellulaire (vésicules de stockage).

adaptatrices APl (adaptor protein 1) et GGA2 (Golgi-associated, gamma adaptin ear-containing, adenosine diphosphate ribosylation factor: ARF-binding protein 2) permettent de recruter la clathrine sur les membranes. De plus, CHC22 s'associe aussi avec des petites protéines de la famille des SNARE (soluble $\mathrm{N}$-ethylmalemide-sensitive factor attachment protein receptor) [15] et en particulier la synaptobrévine (aussi appelée VAMP2) qui est importante pour la fusion des vésicules contenant GLUT4 avec la membrane plasmique. Dans des cultures de cellules musculaires et d'adipocytes humains, CHC22 est colocalisée avec GLUT4 dans ces vésicules. Une diminution de l'expression de CHC22 par siARN induit la perte de ces vésicules et une diminution de l'absorption de glucose en réponse à une stimulation par l'insuline. Inversement, l'expression du gène humain codant pour $\mathrm{CHC2} 2$ chez la souris conduit à une diminution de la quantité de GLUT4 détectée à la membrane plasmique et à une homéostasie anormale du glucose.

\section{CHC22 et diabète de type 2}

L'hypothèse selon laquelle un défaut du trafic membranaire de GLUT4 pourrait contribuer à la pathologie du diabète de type 2 avait été proposée par l'équipe de Timothy Garvey $[7,8]$. Ces deux études montraient que, contrairement à ce qui a déjà été observé pour les modèles de rongeurs diabétiques, même à l'état basal, GLUT4 est anormalement séquestré dans un compartiment intracellulaire dans les cellules musculaires et les adipocytes humains de patients atteints de diabète de type 2 (Figure 2). Nos résultats renforcent cette hypothèse et suggèrent que $\mathrm{CHC} 22$ puisse contribuer à ce défaut. Ceci pourrait être la conséquence soit d'une signalisation de l'insuline défectueuse, l'hormone étant alors incapable d'activer la translocation des vésicules de GLUT4, soit d'un défaut dans le mécanisme de translocation des vésicules. Une hypothèse intéressante serait que, dans les conditions normales, l'insuline induise la perte du manteau de clathrine. L'insuline aurait alors un double effet: d'une part elle inhiberait l'endocytose des transporteurs de surface, et d'autre part elle stimulerait l'export de vésicules qui, dépourvues de leur manteau de clathrine, pourraient alors fusionner librement avec la membrane plasmi- que. Cet effet de l'insuline aurait alors comme conséquence une augmentation drastique des transporteurs présents à la surface. Un défaut dans le démantèlement de la clathrine chez les patients diabétiques aurait pour conséquence l'accumulation de vésicules dans les compartiments intracellulaires. Chez les patients diabétiques, la diminution de Hsc70 (heat shock cognate protein), une protéine chaperone cytosolique qui est impliquée dans le mécanisme de détachement de la clathrine, a d'ailleurs récemment été montrée [9].

\section{Conclusion}

Nos travaux sur CHC22 mettent en évidence pour la première fois des différences dans le mécanisme du trafic membranaire de GLUT4 entre l'humain et la souris. La plupart des études effectuées sur le trafic de GLUT4 ont utilisé des modèles de rongeurs dans lesquels c'est la clathrine $\mathrm{CHCl} 7$ qui permet la formation des vésicules de stockage de GLUT4 [13]. Pourtant, l'intervention dans la formation du compartiment de stockage de GLUT4 humain d'au moins un élément supplémentaire suggère que ce compartiment est plus robuste que le compartiment équivalent 

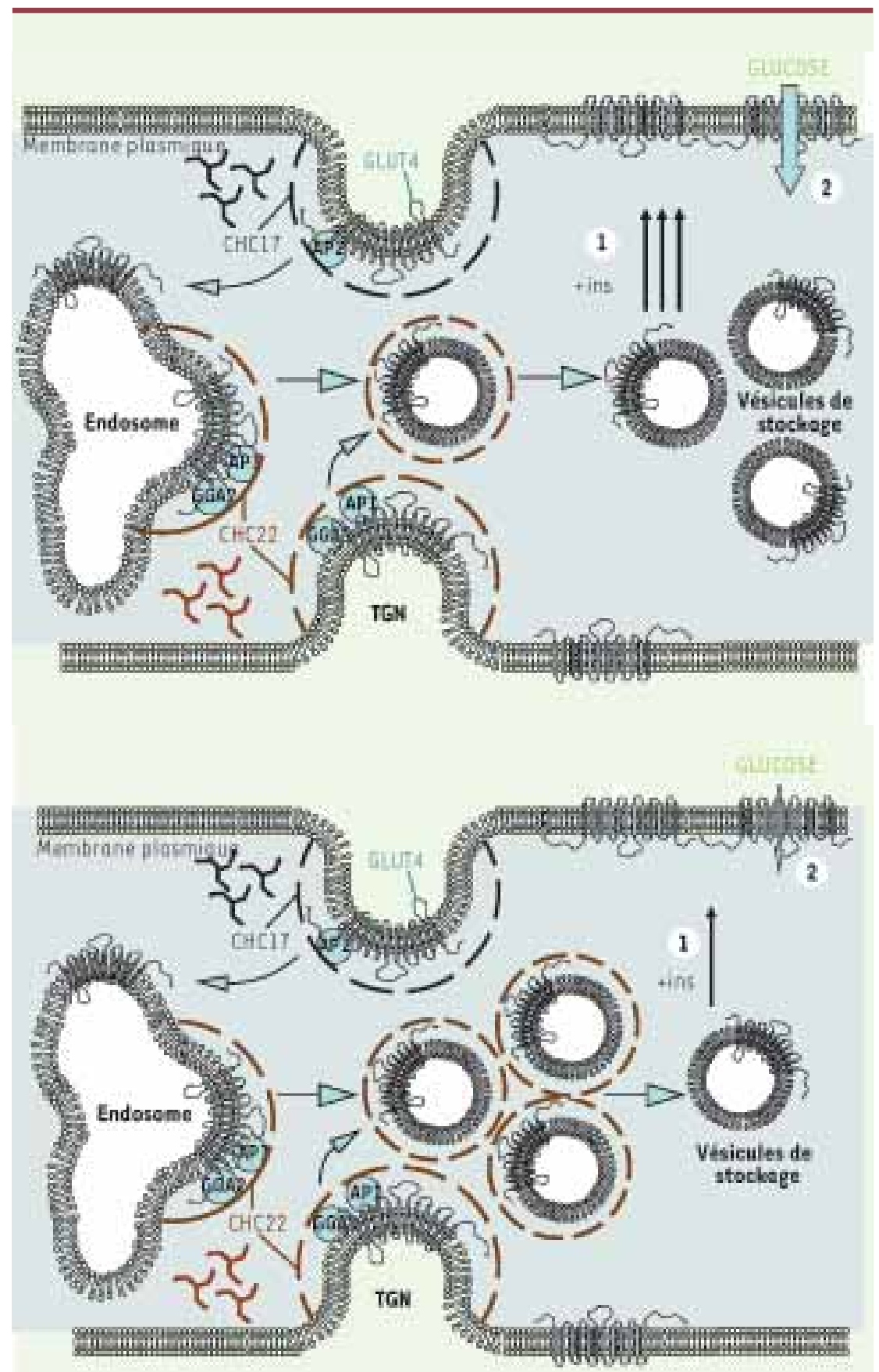

chez la souris, qui est contrôlé par un équilibre entre les voies de biosynthèse et d'endocytose $[10,11]$. Cette différence dans le trafic de GLUT4 entre les muscles humain et murin pourrait contribuer à expliquer certaines différences en termes de métabolisme du glucose. Par exemple, chez l'humain, l'absorption de glucose stimulée par l'insuline dépend en grande
Figure 2. Mouvements des vésicules GLUT4 en réponse à l'insuline. (A) Translocation des vésicules de GLUT4 en réponse à l'insuline et influx de glucose. (1) Chez les individus sains, les GLUT4 stockés dans le compartiment intracellulaire sont transloqués à la membrane plasmique en réponse à l'insuline (+ins). (2) Les transporteurs vont alors permettre l'absorption du glucose sanguin par les cellules musculaires et les adipocytes. (B) Accumulation intracellulaire de vésicules GLUT4 chez les patients diabétiques. Nos travaux soutiennent l'hypothèse selon laquelle GLUT4 est anormalement séquestré dans le compartiment intracellulaire à l'état basal chez ces patients. Cette séquestration pourrait conduire à une diminution du nombre de transporteurs capables d'être transloqués à la surface de la cellule en réponse à l'insuline (+ins (1)) et par conséquent à la diminution de la quantité de glucose sanguin absorbée par les cellules musculaires et les adipocytes (2).

ne sont pas présentes chez les rongeurs et qui pourraient fonctionner dans la même voie de trafic membranaire que CHC22. Le fait que CHC22 soit partiellement capable de fonctionner dans le trafic de GLUT4 chez les souris transgéniques suggère la possibilité de créer un modèle de souris plus complet dans lequel certains aspects spécifiques du métabolisme du glucose humain et du diabète de type 2 pourront être étudiés. $\diamond$

The CHC22 human clathrin heavy chain isoform is involved in the cellular trafic of the glucose transporter GLUT4

\section{CONFLIT D'INTÉRÊTS}

Les auteurs déclarent n'avoir aucun conflit d'intérêts concernant les données publiées dans cet article.

partie du muscle squelettique, alors que chez la souris, le foie est l'organe le plus influent [12]. De plus, notre étude suggère qu'il serait intéressant de rechercher des mutations du gène de $\mathrm{CHC} 22$ chez des patients atteints de diabète de type 2 . Enfin, il est important de souligner qu'il existe chez l'homme d'autres protéines impliquées dans le trafic membranaire qui

\section{RÉFÉRENCES}

1. Huang S, Czech MP. The GLUT4 glucose transporter. Cell Metab $2007 ; 5$ : 237-52.

2. Savage DB, Petersen KF, Shulman GI. Disordered lipid metabolism and the pathogenesis of insulin resistance. Physiol Rev $2007 ; 87$ : 507-20.

3. Shepherd PR, Kahn BB. Glucose transporters and insulin action--implications for insulin resistance and diabetes mellitus. N Engl J Med 1999; 341 : 248-57. 
4. Antonescu CN, Diaz M, Femia G, Planas JV, Klip A. Clathrin-dependent and independent endocytosis of glucose transporter 4 (GLUT4) in myoblasts: regulation by mitochondrial uncoupling. Traffic (Copenhagen, Denmark) 2008 ; 9 : 1173-90.

5. Liu S-H, Towler MC, Chen $\varepsilon$, et al. A novel clathrin homolog that co-distributes with cytoskeletal components functions in the trans-Golgi network. EMBO J $2001 ; 20$ : 272-84

6. Wakeham DE, Abi-Rached L, Towler MC, et al. Clathrin heavy and light chain isoforms originated by independent mechanisms of gene duplication during chordate evolution. Proc Natl Acad Sci USA 2005; $102: 7209-14$

7. Garvey WT, Maianu L, Zhu JH, et al. Evidence for defects in the trafficking and translocation of GLUT4 glucose transporters in skeletal muscle as a cause of human insulin resistance. J Clin Invest 1998 . $101: 2377-86$.

8. Maianu L, Keller SR, Garvey WT. Adipocytes exhibit abnormal subcellular distribution and translocation of vesicles containing glucose transporter 4 and insulinregulated aminopeptidase in type 2 diabetes mellitus: implications regarding defects in vesicle trafficking. J Clin Endocrinol Metab 2001 ; 86 : 5450-6.

9. Chen HS, Jia J, Su HF, et al. Downregulation of the constitutively expressed $\mathrm{Hsc70}$ in diabetic myocardium is mediated by insulin deficiency. J Endocrinol 2006 ; $190: 433-40$

10. Bryant NJ, Govers R, James DE. Regulated transport of the glucose transporter GLUT4. Nat Rev Mol Cell Biol $2002 ; 3: 267-77$.
11. Blot V, McGraw TE. Molecular mechanisms controlling GLUT4 intracellular retention. Mol Biol Cell 2008; $19: 3477-87$.

12. Michael MD, Kulkarni RN, Postic C, et al. Loss of insulin signaling in hepatocytes leads to severe insulin resistance and progressive hepatic dysfunction. Mol Cell $2000 ; 6: 87-97$

13. Fazakerley DJ, Lawrence SP, Lizunov VA, Cushman SW, Holman GD. A common trafficking route for GLUT4 in cardiomyocytes in response to insulin, contraction and energy-status signalling. J Cell Sci 2009 ; 122 : 727-34.

14. Manel N, Kinet S, Kim FJ, et al. GLUT-1 is the receptor of retrovirus HTLV. Med Sci (Paris) 2004 ; $20: 277-9$.

15. Tareste D. Energy of the SNAREpin machinery for membrane fusion. Med Sci (Paris) 2008; 24 : 142-3.
NOUVELLE

\section{La reconnaissance des bactéries de la flore intestinale}

\section{Un important facteur de risque pour la maladie de Crohn}

Alexandra-Chloé Villani, Denis Franchimont

> Contrairement à ce que l'on pensait, la maladie de Crohn ne proviendrait pas initialement d'une réaction inflammatoire excessive dirigée contre la muqueuse intestinale. Elle dépendrait, entre autres, d'une déficience de la réponse immunitaire contre des bactéries pathogènes de la flore intestinale. C'est ce que révèlent des études génétiques portant sur des récepteurs intestinaux reconnaissant ces bactéries.

En général, la flore microbienne intestinale facilite la digestion et l'absorption des nutriments. Toutefois, elle est constamment gardée sous surveillance par le système immunitaire. Celui-ci doit pouvoir la tolérer tout en restant capable de reconnaître les agents pathogènes et de se défendre contre eux lors d'attaques épisodiques. Tout déficit de reconnaissance ou toute altération de cet équilibre peut mener à l'apparition de maladies inflammatoires du système digestif comme la maladie de Crohn $[1,2]$.

Maladie de Crohn, I'hypothèse initiale pro-inflammatoire La maladie de Crohn est une maladie inflammatoire chronique d'origine multifactorielle et hétérogène affectant aurait pour origine une réponse inappropriée du système immunitaire digestif contre la flore intestinale chez des individus génétiquement prédisposés [2]. Cette maladie se manifeste par une inflammation de la muqueuse intestinale associée à de petites ulcérations aphtoïdes ou de larges ulcères au sein du tube digestif. Ces derniers sont responsables de deux complications: l'apparition de rétrécissements de l'intestin grêle (ou sténoses) et de perl'ensemble du tube digestif [2]. Elle
A.C. Villani : département

de gastroentérologie, Centre universitaire de santé McGill, Hôpital général de Montréal, 1650, avenue Cedar, Montréal (Québec)

H3G 1A4, Canada;

Centre d'innovation Génome Québec

et Université McGill,

740, avenue du Docteur-Penfield, Montréal (Québec) H3A 1A4, Canada. alexandra-chloe.villani@mail.mcgill.ca

D. Franchimont : département

de gastroentérologie, Centre universitaire de santé McGill, Hôpital général de Montréal, 1650, avenue Cedar, Montréal (Québec)

H3G IA4, Canada;

département de gastroentérologie, Hôpital Érasme, ULB,

808, chemin Lennik, 1070 Bruxelles, Belgique.

forations de la paroi intestinale ou de fistules et d'abcès intra-abdominaux. Ces symptômes résultent d'une infiltration massive de cellules inflammatoires dans la paroi intestinale. Les cytokines proinflammatoires qu'elles produisent sont responsables de l'activation et de la propagation de la réponse immunitaire innée et acquise [2]. Ces observations ont initialement fait penser que la maladie de Crohn résultait d'une réponse inflammatoire agressive et excessive, caractérisée entre autres par une surexpression et une surproduction incontrôlée de médiateurs proinflammatoires. L'utilisation bénéfique 